\title{
Prevalence of Metabolic Syndrome and Related Factors in Patients with Non-alcoholic Fatty Liver
}

\author{
Mehrnoosh Zakerkish ${ }^{1,}{ }^{*}$, Abolghasem Assarzadeh ${ }^{1}$, Seyed Saeed Seyedian ${ }^{2}$ and Alireza Jahanshahi ${ }^{1}$ \\ ${ }^{1}$ Health Research Institute, Diabetes Research Center, Ahvaz Jundishapur University of Medical Sciences, Ahvaz, Iran \\ ${ }^{2}$ Department of Gastroenterology, School of Medicine, Ahvaz Jundishapur University of Medical Sciences, Ahvaz, Iran \\ "Corresponding author: Health Research Institute, Diabetes Research Center, Ahvaz Jundishapur University of Medical Sciences, Ahvaz, Iran. Email: zakerkish-m@ajums.ac.ir
}

Received 2021 August 24; Revised 2021 November 09; Accepted 2021 November 10.

\begin{abstract}
Objectives: This study was designed to investigate the prevalence of metabolic syndrome in patients diagnosed with non-alcoholic fatty liver disease (NAFLD) referring to the gastrointestinal and endocrinology clinics of Golestan and Imam Khomeini Hospitals, Ahvaz.

Methods: The current cross-sectional study was performed on patients with NAFLD referring to the gastroenterology and endocrinology clinics of Golestan and Emam Khomeini hospitals, Ahvaz in the second half of 2020. Demographic information included age, gender, lumbar posture (while standing and the waist be in the upper edge of the iliac crest at the end of a normal exhalation using a non-elastic meter), grading fatty liver evidenced by ultrasound, and blood pressure and laboratory parameters, including triglyceride (TG), fasting blood sugar (FBS), and high-density lipoprotein (HDL) was recorded in the checklist. Data analysis was performed using SPSS 26.0 software.

Results: This study was performed on 130 patients with an average age of $46.22 \pm 14.27$ years. Patients comprised 42 men (32.8\%) and 88 women (67.2/\%). Also, $63.84 \%$ of the patients with NAFLD had metabolic syndrome, and 56.2, 69.6, and $81.8 \%$ with grades 1,2 , and 3 of fatty liver, respectively had metabolic syndrome. There was a significant difference in terms of mean height and weight in both gender. There was a significant relationship between age and FBS, so that the levels of FBS increased statistically significantly with age $(\mathrm{P}<0.05)$. Gender had a statistically significant relationship with HDL and waist circumference $(\mathrm{P}=0.038)$. There was no statistically significant relationship between blood pressure, TG, HDL, FBS, and waist circumference, and different grades of fatty liver $(\mathrm{P}>0.05)$.

Conclusions: The current study showed a significant number of patients with NAFLD had metabolic syndrome, so that the most common symptom of metabolic syndrome was low HDL levels, followed by high FBS levels, blood pressure, and TG levels and waist circumference size. Nevertheless, it was not probable to assess whether NAFLD precedes the progress of metabolic syndrome.
\end{abstract}

Keywords: Non-alcoholic Fatty Liver Disease, Metabolic Syndrome, Waist Circumference, Hyperglycemia

\section{Background}

The liver is a critical organ in the human body that is responsible for several physiological processes, such as making proteins, blood clotting factors, metabolism of carbohydrates, lipids, and proteins, blood volume maintenance, boosting the immune system, regulation of the growth hormone receptor signaling pathway, homeostasis of cholesterol and lipid, glycogen synthesis, bile production, and the breakdown of xenobiotic compounds (1). Non-alcoholic liver disease (NAFLD) refers to liver abnormalities, including simple steatosis or non-alcoholic fatty liver to non-alcoholic steatohepatitis (NASH) with or without cirrhosis progress (2). The current meaning of NAFLD does not need secondary hepatic fat accumulation, such as significant alcohol consumption, use of steatogenic medication, or hereditary disorders (3). Numerous studies have independently shown a robust relationship between NAFLD and each feature of metabolic syndrome (MetS). All guidelines currently approve that NAFLD is stringently linked to metabolic risk factors, particularly obesity, type 2 diabetes mellitus (T2DM), and dyslipidemia. However, it has been recommended that NAFLD shares many features of the MetS $(2,4)$.

NAFLD is one of the most common chronic liver diseases and is a term used to describe all diseases associated with the accumulation of too much fat in the liver cells (5). NAFLD progresses slowly and can develop into liver cirrhosis, liver failure, and hepatocellular carcinoma (HCC). NAFLD is the term for a range of mild liver disease caused 
by a build-up of fat in the liver that leads to a chronic and irreversible disease called cirrhosis (6). In a group of fatty liver patients similar to those who consume alcohol, liver cell damage occurs, but in these patients, there is no history of alcohol consumption. There was no evidence of other liver cell diseases in these patients, but it was observed that $82 \%$ of them were obese, $50 \%$ of them had hyperlipidemia, and $50 \%$ were diabetics (7). Based on the sensitivity of the imaging method, the incidence and prevalence NAFLD varies in different studies. In most cases, the disease is asymptomatic and is detected accidentally by observing high levels of liver enzymes in blood tests or abdominal ultrasound performed for other reasons (8). However, some patients rarely complain of vague pain in the upper and right abdomen or a feeling of premature fatigue (9). Obesity is one of the most important diseases associated with fatty liver. Body mass index (BMI) and waist circumference (WC) are widely used anthropometric measures to assess the effects of obesity on risk factors for MetS and NAFLD (10). Unlike WC and BMI, waist-to-hip ratio (WHR) and waist-to-height ratio (WHtR) values have advantages without the need for population-specific reference tables or changes in body composition with growth and development. Therefore, WHR is more strongly related to abdominal obesity than BMI, and WHR is strongly related to visceral adipose tissue (VAT) (11). Hyperlipidemia is another factor of fatty liver disease, and appropriate treatment of hyperlipidemia leads to a reduction in the process of destruction of liver cells in fatty liver disease (12). MetS is as a concordance of cardiovascular risk factors associated with obesity, including abdominal obesity, impaired glucose intolerance, hypertriglyceridemia, low high-density lipoprotein (HDL) cholesterol, and/or hypertension (13). Patients with this syndrome have an enhanced risk of developing cardiovascular disease and/or type 2 diabetes. Obesity and inactivity are the major environmental factors to develop MetS (14). Numerous factors cause MetS, especially insulin resistance, adipose tissue dysfunction, chronic inflammation, oxidative stress, circadian disruption, microbiota, genetic factors, maternal programming, rapid urbanization, nutritional factors, inactivity, social, economic, and cultural factors, and psychosocial stresses (15). Although the underlying physiopathological cause of this syndrome is unknown, strong evidence suggests that insulin resistance is the leading cause of MetS (16). The definition of NCEP ATP III is one of the most extensively used criteria for MetS. The NCEP/ATP III criteria for diagnosing MetS are as follows (diagnosed when three or more are present): (1) WC greater than 40 inches (males) or 35 inches (females); (2) blood pressure greater than 130/85 mmHg; (3) fasting triglyceride (TG) levels greater than 150 $\mathrm{mg} / \mathrm{dL}$; (4) fasting HDL cholesterol level $40 \mathrm{mg} / \mathrm{dL}$ (men) or
$50 \mathrm{mg} / \mathrm{dL}$ (women); and (5) fasting blood sugar (FBS) more than $100 \mathrm{mg} / \mathrm{dL}(17)$.

\section{Objectives}

The purpose of this study was to assess the frequency of MetS in patients with NAFLD referring to the gastroenterology and endocrinology clinics of Golestan and Emam Khomeini hospitals of Ahvaz in 2021.

\section{Methods}

The current research was an analytical cross-sectional study conducted on patients with fatty liver referring to the gastroenterology and endocrinology clinics of Golestan and Emam Khomeini hospitals of Ahvaz in the second half of 2020. According to a study by Fattahi et al. (18) and considering the prevalence rate of $65 \%$, the sample size was calculated based on the following formula:

$n=\frac{\left(z_{1-\frac{\alpha}{2}}+z_{1-\beta}\right)^{2} p q}{d^{2}}=\frac{(1.96+1.64)^{2} 0.65 \times 0.35}{(0.15)^{2}}=130$

A non-probability convenient sampling method was used. In this study, all the provisions of the ethics statement in Helsinki Declaration and the principles of confidentiality of patient information were considered. Inclusion criteria were the age of over 18 years and diagnosis non-alcoholic fatty liver based on ultrasound. Exclusion criteria included alcohol and drug users, users of drugs with liver side effects, such as amiodarone and tamoxifen, pregnant women, patient with known liver disease, and a positive history for HCV, HBC, or HIV, and history of cirrhosis and other liver diseases. After confirming the inclusion and non-exclusion criteria, NAFLD patients were selected, and all demographic information, including age, gender, lumbar posture (patient standing and waist in the upper edge of the iliac crest at the end of a normal exhalation using a non-elastic meter), grading fatty liver by ultrasound, as well as the patients' blood pressure was extracted from the patients' records in the hospital and written in the checklist. Then patients were referred to the laboratory for a blood test (after 12 hours of fasting) to assess TG, FBS, and HDL. In the end, clinical and demographic characteristics of the patients were recorded and statistically analyzed.

\subsection{Ethics}

This study was approved by the Ethics Committee of the Jundishapur University of Medical Sciences, Ahwaz (IR.AJUMS.HGOLESTAN.REC.1399.135). Each patient signed the informed consent. 


\subsection{Statistical Analysis}

The data analysis was carried out by SPSS Version 22 (IBM). The normal distribution of the quantitative data was checked using the Kolmogorov-Smirnov test. The relationship between qualitative variables was assessed by chisquare or Fisher's exact tests, while the Pearson correlation coefficient was used to assess the relationship between quantitative variables. Quantitative values were compared between the two groups using an independent $t$-test and more than two groups using one-way analysis of variance (ANOVA) or its nonparametric equivalent. A P-value $<0.05$ was considered statistically significant.

\section{Results}

This study was performed on 130 patients with an average age of $46.22 \pm 14.27$ years. Patients were 42 men (32.8\%) and 88 women (67.2/\%). Descriptive characteristics of the study variables are presented in Table 1.

\begin{tabular}{|c|c|}
\hline Variables & Values \\
\hline Age & $46.22 \pm 14.27$ \\
\hline \multicolumn{2}{|l|}{ Gender } \\
\hline Woman & $88(67.2)$ \\
\hline Men & $42(32.8)$ \\
\hline \multicolumn{2}{|c|}{ Non-alcoholic fatty liver grading } \\
\hline Grade 1 & $73(56.2)$ \\
\hline Grade 2 & $46(35.4)$ \\
\hline Grade 3 & $11(8.5)$ \\
\hline Weight & $88.67 \pm 19.00$ \\
\hline Height & $165.11 \pm 13.12$ \\
\hline BMI & $31.78 \pm 6.60$ \\
\hline Waist & $105.54 \pm 17.24$ \\
\hline BP & $132.74 \pm 20.14$ \\
\hline FBS & $123.14 \pm 48.25$ \\
\hline TG & $223.12 \pm 103.66$ \\
\hline HDL & $49.22 \pm 39.31$ \\
\hline
\end{tabular}

${ }^{\mathrm{a}}$ Values are expressed as mean $\pm \mathrm{SD}$ or No. (\%).

The chi-square test showed that there was a significant difference between gender and fatty liver. Men are more at the risk for grade 2 , and women are more at grade 1 of fatty liver. Patients with grade 3 were older than the other two grades. There was a statistically significant difference between age groups based on fatty liver grade $(\mathrm{P}=0.026$,
Table 2), while there was not a statistically significant difference between BMI groups based on fatty liver rating $(\mathrm{P}=$ 0.98, Table 3).

Women had a longer history of diabetes than men. But the mean age in the diabetic group was significantly higher than the non-diabetic group $(\mathrm{P}=0.002)$.

There was a statistically significant difference between the two genders in terms of mean height and weight, while other variables had statistically equal means in both genders. Men's weight and height were significantly higher than women. There was a significant difference between age and FBS where the level of FBS increased significantly with age. It was also obvious that height decreased with age.

The results of chi-square test showed a statistically significant between both genders regarding HDL and WC (P $=0.038$ ). The t-test results showed that the mean age was significantly higher in patients with FBS below $100 \mathrm{mg} / \mathrm{dL}$ and blood pressure over $130 \mathrm{~mm} \mathrm{Hg}$. Also, the ANOVA results showed a significant age difference between patients with different degrees of MetS $(\mathrm{P}<0.05)$. The relationship between between gender and MetS markers is shown in Table 4 .

There was no statistically significant difference between BP, TG, HDL, FBS, and WC and different grades of fatty liver $(P>0.05)$. No significant difference was observed between MetS and fatty liver grade $(\mathrm{P}>0.05)$. However, in grades 1 and 2, the number of patients with MetS was higher and in grade 3, all patients had MetS (Table 5).

In men, there is no statistically significant difference between fatty liver grade and MetS and its markers ( $\mathrm{P}>$ 0.05). In women, however, there was a statistically significant difference between fatty liver grade and MetS and its markers $(\mathrm{P}<0.05)$.

The results of this study showed that the history of diabetes was higher in women than men, but this difference was not statistically significant $(\mathrm{P}=0.761)$. The mean age in the diabetic group was significantly higher than the nondiabetic group $(\mathrm{P}=0.02)$.

\section{Discussion}

In this study, $63.84 \%$ of the patients with NAFLD had MetS. Also, $56.2 \%$ of patients with grade 1 fatty liver had MetS, and $54.8 \%$ of patients with grade 1 fatty liver had high blood pressure, 58.9\% had high FBS, 32.9\% had high TG levels (above 150), 57.5\% had low HDL, and 19.2\% had high WC. In addition, $69.6 \%$ of patients with grade 2 fatty liver had MetS. In patients with grade 2 fatty liver, $43.5 \%$ had high blood pressure, 54.3\% had high blood sugar, $15.2 \%$ had high TG levels, 56.5\% had low HDL, and 73.9\% had high WC. Also, 
Table 2. The Relationship Between Age and Fatty Liver Grade

\begin{tabular}{lcccc}
\hline Variables & No. & Mean \pm SD & Min & Pax \\
\hline Grade 1 & 73 & $47.2740 \pm 15.12564$ & 17.00 & 79.00 \\
Grade 2 & 46 & $43.7826 \pm 13.29146$ & 20.00 & 75.00 \\
Grade 3 & 11 & $49.3636 \pm 12.45209$ & 29.00 & 6.02606 \\
Total & 130 & $46.2154 \pm 14.31538$ & 17.00 & 79.00 \\
\hline
\end{tabular}

Table 3. The Relationship Between BMI and Fatty Liver Grade

\begin{tabular}{|c|c|c|c|c|c|}
\hline Variables & No. & Mean $\pm S D$ & Min & Max & P-Value \\
\hline Grade 1 & 73 & $31.44 \pm 9.79$ & 20.32 & 96.12 & \multirow{4}{*}{0.98} \\
\hline Grade 2 & 46 & $33.61 \pm 7.64$ & 21.36 & 52.69 & \\
\hline Grade 3 & 11 & $33.55 \pm 5.56$ & 25.65 & 46.14 & \\
\hline Total & 130 & $31.78 \pm 6.60$ & 20.32 & 96.12 & \\
\hline
\end{tabular}

Table 4. The Relationship Between Gender and Different Levels of Metabolic Syndrome Markers ${ }^{\text {a }}$

\begin{tabular}{|c|c|c|c|}
\hline Variables & Woman & Men & P-Value \\
\hline BP & & & 0.464 \\
\hline Above 130 & $45(51.1)$ & $19(44.2)$ & \\
\hline Under 130 & $43(48.9)$ & $24(55.8)$ & \\
\hline FBS & & & 0.852 \\
\hline Above 100 & $50(56.8)$ & $23(53.5)$ & \\
\hline Under 100 & $38(43.2)$ & $20(46.5)$ & \\
\hline TG & & & 0.999 \\
\hline Above 150 & $22(25.0)$ & $11(25.6)$ & \\
\hline Under 150 & $66(75.0)$ & $32(74.4)$ & \\
\hline HDL & & & 0.038 \\
\hline Above $50 / 40$ & $31(35.2)$ & $24(55.8)$ & \\
\hline Under 50/40 & $57(64.8)$ & $19(44.2)$ & \\
\hline Waist & & & $<0.001$ \\
\hline Above 102/88 & $10(11.4)$ & $18(41.9)$ & \\
\hline Under 102/88 & $78(88.6)$ & $25(58.1)$ & \\
\hline Number of symptoms metabolic syndrome & & & 0.678 \\
\hline 0 & $1(1.1)$ & $2(4.7)$ & \\
\hline 1 & $9(10.2)$ & $6(14.0)$ & \\
\hline 2 & $19(21.6)$ & $11(25.6)$ & \\
\hline 3 & $21(23.9)$ & $10(23.3)$ & \\
\hline 4 & $18(20.5)$ & $8(18.6)$ & \\
\hline 5 & $20(22.7)$ & $6(14.0)$ & \\
\hline Metabolic syndrome & & & 0.248 \\
\hline No & $29(33.0)$ & $19(44.2)$ & \\
\hline Yes & $59(67.0)$ & $24(55.8)$ & \\
\hline
\end{tabular}

${ }^{\text {a }}$ Values are expressed as No. (\%). 


\begin{tabular}{|c|c|c|c|c|}
\hline Variables & Grade 1 & Grade 2 & Grade 3 & P-Value \\
\hline BP & & & & 0.367 \\
\hline Above 130 & $40(54.8)$ & $20(43.5)$ & $4(36.4)$ & \\
\hline Under 130 & $33(45.2)$ & $26(56.5)$ & $7(63.6)$ & \\
\hline FBS & & & & 0.661 \\
\hline Above 100 & $43(58.9)$ & $25(54.3)$ & $5(45.5)$ & \\
\hline Under 100 & $30(41.1)$ & $21(45.7)$ & $6(54.5)$ & \\
\hline TG & & & & 0.097 \\
\hline Above 150 & $24(32.9)$ & $7(15.2)$ & $2(18.2)$ & \\
\hline Under 150 & $49(67.1)$ & $39(84.8)$ & $9(81.8)$ & \\
\hline HDL & & & & 0.929 \\
\hline Above 50/40 & $31(42.5)$ & $20(43.5)$ & $4(36.4)$ & \\
\hline Under 50/40 & $42(57.5)$ & $26(56.5)$ & $7(63.6)$ & \\
\hline Waist & & & & 0.690 \\
\hline Above $102 / 88$ & $14(19.2)$ & $12(26.1)$ & $2(18.2)$ & \\
\hline Under 102/88 & $59(80.8)$ & $34(73.9)$ & $9(81.8)$ & \\
\hline Number of symptoms metabolic syndrome & & & & 0.398 \\
\hline 0 & $2(2.7)$ & $1(2.2)$ & $0(0.0)$ & \\
\hline 1 & $9(12.3)$ & $5(10.9)$ & $1(9.1)$ & \\
\hline 2 & $21(28.8)$ & $8(17.4)$ & $1(9.1)$ & \\
\hline 3 & $14(19.2)$ & $12(26.1)$ & $5(45.5)$ & \\
\hline 4 & $15(20.5)$ & $11(23.9)$ & $0(0.0)$ & \\
\hline 5 & $12(16.4)$ & $9(19.6)$ & $4(36.4)$ & \\
\hline Metabolic syndrome & & & & 0.141 \\
\hline No & $32(43.8)$ & $14(30.4)$ & $2(18.2)$ & \\
\hline Yes & $41(56.2)$ & $32(69.6)$ & $9(81.8)$ & \\
\hline
\end{tabular}

$81.8 \%$ of patients with grade 3 fatty liver had MetS. In patients with grade 3 fatty liver, $36.4 \%$ had high FBS, $45.5 \%$ had high TG levels, 18.2 - 63.6\% had low HDL levels, and 81.8\% had high WC. There was a significant difference between gender and fatty liver. The results showed a significant difference between age and three grades of fatty liver. There was a significant difference in terms of mean height and weight in both genders. Also, there was a statistically significant difference between both genders regarding HDL and WC. In women, however, there was a statistically significant difference between fatty liver grade and MetS and its markers.

Further studies have shown the rate of MetS to be 10 - 30\% in Taiwan, Japan, India, the United States, Europe, and Indonesia. Furthermore, the prevalence of MetS in NAFLD patients ranged from 61 to $65 \%$ according to CCDMIA and NCEP/ATPIII, respectively, which was 1.8 - 3.1 times higher than in previous reports. This is moderately higher than that reported by Uchil et al. among Indian adult patients with NAFLD (47\%) (19). In general, the prevalence of MetS varied significantly between studies depending on the population studied and the diagnostic criteria used (18).

In the study by Fakhrzadeh et al. (2004), the raw prevalence of MetS in the study population was $29.9 \%$ and the most common metabolic disorders were high TG levels and high blood pressure, followed by high WC, low HDL levels, and high FBS. In the mentioned study, the only criterion that had a significant relationship with gender was WC, which was significantly more common in women, while in our study, high WC and low HDL levels had a significant relationship with gender (20). In this study, there was a significant relationship between blood pressure (above 130 $\mathrm{mm} \mathrm{Hg}$ and below $130 \mathrm{~mm} \mathrm{Hg}$ ) and high FBS and age, but 
in the present study, the only variable related to age was an increase in FBS. The mean age of patients with no symptoms of MetS was 24.33 years, and the mean age of those who had all five symptoms of MetS was 51.80. Finally, in term of age, there was no statistically significant difference between patients with MetS than patients without MetS, while in our study, the only age-related variable was high FBS. Also, there was no statistically significant difference between patients regarding weight at different ages, but there was a significant difference in height and BMI at different ages. FBS and HDL levels were significantly different at different ages. Other factors were not significantly associated with age.

In the present study, regarding the history of underlying disease in patients based on age, it was vary according to the age of patients. There was no significant difference between patients in terms of the age of onset of high blood lipids. There was a statistically significant relationship between the drugs used for diabetes and having diabetes at different ages.

Amiri and Hakimi (21) showed that the prevalence of MetS among nurses in Bandar Abbas was 11.67\%. However, in different studies, this percentage is very different. Therefore, it can be said that different prevalence rates of MetS in different regions can be due to different lifestyles, eating patterns, and environmental and genetic factors. In the mentioned study, the prevalence of MetS was higher in men than women, so that its prevalence was $23.52 \%$ in men and $10.55 \%$ in women. They indicated that MetS had no significant relationship with age, gender, type of job, and work shift and reported only a significant relationship between MetS and BMI.

A meta-analysis by Maleki et al. reported that at least one-fifth of the population of Iran have MetS, and the overall prevalence of MetS in Iran was 21.1\% (22). It reported a lower rate compared to the prevalence of MetS in the United States (34\%) as well as some European countries (23\%). But the prevalence of MetS in Iran was higher compared to East Asia. They demonstrated that the prevalence of MetS is higher in women than men, but this difference was no statistically significant. Mabry et al. (23) reported that the prevalence of MetS was higher in women compared to men in some countries of the Persian Gulf.

Recently, Paudel et al. (2019) assessed the prevalence MetS in 385 patients with NAFLD based on NCEP-ATPIII criteria and reported it to be $57.6 \%$. They showed that at least one component of MetS was found in $91.4 \%$ of patients with radiological features of fatty liver. The largest proportion of NAFLD patients were men. Increased lumbar circumference after HDL levels were the most common component of MetS in NAFLD patients (24).

However, a similar study in Australia reported a higher prevalence of MetS in men than women (25). In our study, the higher prevalence of MetS in women with fatty liver disease may be due to the greater prevalence of obesity and inactivity in Iranian women. In addition, most of the patients who had been referred to this hospital were possibly unemployed women. They spend longer periods of time at home and consequently, eat more foods and are less active. Consistent with our results, a review study demonstrated that the greatest condition in the studied population was the low HDL cholesterol levels (prevalence: 59.7\%). Low HDL cholesterol levels in Iran may be related to lifestyle patterns, such as unhealthy diet, physical inactivity, genetic factors, such as mutations in the CETP locus, and increased hepatic lipase production. Also, in this review study, the second most frequent condition was hypertriglyceridemia followed by central obesity and hypertension. The prevalence of FBS in this study was the lowest among the components of MetS, which contradicts with our results.

Mazloomzadeh et al. (2018) in Iran showed that the prevalence of MetS increases with age. In this study, the prevalence of the syndrome was $23.8 \%$ in patients over 20 years of age and $10.9 \%$ in those under 20 years of age, which was different from the results of our study because, in term of age, there was no statistically significant difference between patients with MetS compared to patients without MetS (26). Ervin in the United States (2009) showed that $34 \%$ of adults had MetS. On the other hand, he reported that the probability of MetS increases with age and BMI, but race and ethnicity had no significant effect on the prevalence of MetS (27). Patton et al. in the United States (2011) reported that the prevalence of MetS was 25.6\%. Also, the most common symptoms were central obesity and hypertension, which contradicted the results of the present study. In this study, they indicated that only $13.5 \%$ of children had no feature of MetS, and the majority of them (77.2\%) had one to three features of this syndrome (28). The potential cause of fatty liver disease and MetS may be insulin resistance or oxidative stress. Mechanisms of action of the insulin are one of hypotheses that leads to TG accumulation in the liver cells $(29,30)$. Peripheral insulin resistance leads to an increase in insulin levels in the blood, which by regulating lipogenic factors and increasing the entry of free fatty acids into the liver due to the loss of insulin-sensitive lipase inhibition, increases the entry and invasion of free fatty acids into the liver cells $(31,32)$. The prevalence of MetS in different studies varies according to the studied samples and the widely used diagnostic criteria. Fattahi et al. (2016) in Iran showed no difference between men and women in terms of the prevalence of MetS in patients with NAFLD, but the prevalence of NAFLD was higher in men than women, which contradicts the results of the present study. The prevalence of NAFLD in this study 
was $25.7 \%$, and the prevalence of MetS in these patients was reported to be 61 - 65\%. In this study, hyperglycemia and abdominal obesity were very common in patients with NAFLD and MetS (18). Numerous studies have shown that MetS and insulin resistance are highly involved in NAFLD $(7,33,34)$.

\subsection{Conclusions}

The present study indicated a considerable number of patients with NAFLD had MetS so that the most common symptom of MetS was low HDL levels, followed by high FBS levels, high blood pressure, and high TG levels, and high WC size. Nevertheless, it was not possible to determine whether NAFLD predates the development of MetS.

\section{Footnotes}

Authors' Contribution: Study concept and design, F. M., and H. E.; Analysis and interpretation of data, F. M., and S. B.; Drafting of the manuscript, F. M.; Critical revision of the manuscript for important intellectual content, F. M., H. E., and S. B.; Statistical analysis, H. E.

Conflict of Interests: No potential conflicts of interests relevant to this article were reported.

Ethical Approval: This study was approved by the Ethics Committee of the Jundishapur University of Medical Sciences, Ahwaz (IR.AJUMS.HGOLESTAN.REC.1399.135)

Funding/Support: This article is extracted from the thesis in internal medicine residency with registration number RDC-9924. This study was supported by the Jundishapur University of Medical Sciences, Ahvaz, Iran.

Informed Consent: Each patient signed the informed consent.

\section{References}

1. Hoekstra LT, de Graaf W, Nibourg GA, Heger M, Bennink RJ, Stieger B, et al. Physiological and biochemical basis of clinical liver function tests: a review. Ann Surg. 2013;257(1):27-36. doi: 10.1097/SLA.0b013e31825d5d47. [PubMed: 22836216].

2. Streba LA, Vere CC, Rogoveanu I, Streba CT. Nonalcoholic fatty liver disease, metabolic risk factors, and hepatocellular carcinoma: an open question. World J Gastroenterol. 2015;21(14):4103-10. doi: 10.3748/wjg.v21.114.4103. [PubMed: 25892859]. [PubMed Central: PMC4394070].

3. Chalasani N, Younossi Z, Lavine JE, Diehl AM, Brunt EM, Cusi K, et al. The diagnosis and management of non-alcoholic fatty liver disease: practice Guideline by the American Association for the Study of Liver Diseases, American College of Gastroenterology, and the American Gastroenterological Association. Hepatology. 2012;55(6):2005-23. doi: 10.1002/hep.25762. [PubMed: 22488764].

4. Troisi J, Belmonte F, Bisogno A, Pierri L, Colucci A, Scala G, et al. Metabolomic Salivary Signature of Pediatric Obesity Related Liver Disease and Metabolic Syndrome. Nutrients. 2019;11(2) doi: 10.3390/nu11020274. [PubMed: 30691143]. [PubMed Central: PMC6412994]
5. Al-Dayyat HM, Rayyan YM, Tayyem RF. Non-alcoholic fatty liver disease and associated dietary and lifestyle risk factors. Diabetes Metab Syndr. 2018;12(4):569-75. doi: 10.1016/j.dsx.2018.03.016. [PubMed: 29571977].

6. Ahmed M. Non-alcoholic fatty liver disease in 2015. World J Hepatol. 2015;7(11):1450-9. doi: 10.4254/wjh.v7.i11.1450. [PubMed: 26085906]. [PubMed Central: PMC4462685].

7. Paschos P, Paletas K. Non alcoholic fatty liver disease and metabolic syndrome. Hippokratia. 2009;13(1):9.

8. Santos LF, Hernández G, Puerta AV, Beltrán Ó, Botero RC, Mejía G. Enfermedad hepática por infiltración grasa no alcohólica. La nueva pandemia del milenio. Rev Colomb Gastroenterol. 2010;25(4):380-98.

9. Sweet PH, Khoo T, Nguyen S. Nonalcoholic Fatty Liver Disease. Prim Care. 2017;44(4):599-607. doi: 10.1016/j.pop.2017.07.003. [PubMed: 29132522].

10. Tahir F, Majid Z, Majid B, Ahmed J, Zaman A, Tariq M, et al. Anthropometric Measurements and Lifestyle Characteristics of Individuals with Non-alcoholic Fatty Liver Disease. Cureus. 2020;12(2). e7016. doi: 10.7759/cureus.7016. [PubMed: 32211252]. [PubMed Central: PMC7081744].

11. Lin MS, Lin TH, Guo SE, Tsai MH, Chiang MS, Huang TJ, et al. Waistto-height ratio is a useful index for nonalcoholic fatty liver disease in children and adolescents: A secondary data analysis. BMC Public Health. 2017;17(1):851. doi: 10.1186/s12889-017-4868-5. [PubMed: 29084519]. [PubMed Central: PMC5663116].

12. Kneeman JM, Misdraji J, Corey KE. Secondary causes of nonalcoholic fatty liver disease. Therap Adv Gastroenterol. 2012;5(3):199-207. doi: 10.1177/1756283X11430859. [PubMed: 22570680]. [PubMed Central: PMC3342568].

13. Tune JD, Goodwill AG, Sassoon DJ, Mather KJ. Cardiovascular consequences of metabolic syndrome. Transl Res. 2017;183:57-70. doi: 10.1016/j.trsl.2017.01.001. [PubMed: 28130064]. [PubMed Central: PMC5393930].

14. Alshehri AM. Metabolic syndrome and cardiovascular risk. J Family Community Med.2010;17(2):73-8. doi:10.4103/1319-1683.71987. [PubMed: 21359028]. [PubMed Central: PMC3045098].

15. Xu H, Li X, Adams H, Kubena K, Guo S. Etiology of Metabolic Syndrome and Dietary Intervention. Int J Mol Sci. 2018;20(1). doi: 10.3390/ijms20010128. [PubMed: 30602666]. [PubMed Central: PMC6337367].

16. Roberts CK, Hevener AL, Barnard RJ. Metabolic syndrome and insulin resistance: Underlying causes and modification by exercise training. Compr Physiol. 2013;3(1):1-58. doi: 10.1002/cphy.c110062. [PubMed: 23720280]. [PubMed Central: PMC4129661].

17. Moy FM, Bulgiba A. The modified NCEP ATP III criteria maybe better than the IDF criteria in diagnosing Metabolic Syndrome among Malays in Kuala Lumpur. BMC Public Health. 2010;10:678. doi: 10.1186/1471-2458-10-678. [PubMed: 21054885]. [PubMed Central: PMC2989964].

18. Fattahi MR, Niknam R, Safarpour A, Sepehrimanesh M, Lotfi M. The Prevalence of Metabolic Syndrome In Non-alcoholic Fatty Liver Disease; A Population-Based Study. Middle East J Dig Dis. 2016;8(2):1317. doi: 10.15171/mejdd.2016.18. [PubMed: 27252820]. [PubMed Central: PMC4885612].

19. Uchil D, Pipalia D, Chawla M, Patel R, Maniar S; Narayani, et al. Non-alcoholic fatty liver disease (NAFLD)-the hepatic component of metabolic syndrome. J Assoc Physicians India. 2009;57:201-4. [PubMed: 19588647].

20. Fakhrzadeh H, Ebrahimpour P, Nouri M, Pour-Ebrahim R, Heshmat $\mathrm{R}$, Shoushtarizadeh P. [Evaluation of prevalence of the metabolic syndrome in inhabitants of Tehran university of medical sciences population lab]. J Diabetes Metab Disord. 2004;3(Supl 1):71-80. Persian.

21. Amiri A, Hakimi A. [The study of prevalence of metabolic syndrome among nurses of Shahid Mohammadi Hospital of Bandar Abbas city, Iran]. J Clin Nurs Mid. 2017;6(1):1-8. Persian. 
22. Maleki F, Sayehmiri F, Kiani F, Sayehmiri K, Nasiri S. [Metabolic syndrome prevalence in Iran: a systematic review and meta-analysis]. J Kermanshah Univ Med Sci. 2014;18(4). Persian. e74115.

23. Mabry RM, Reeves MM, Eakin EG, Owen N. Gender differences in prevalence of the metabolic syndrome in Gulf Cooperation Council Countries: A systematic review. Diabet Med. 2010;27(5):593-7. doi: 10.1111/j.1464-5491.2010.02998.x. [PubMed: 20536958].

24. Paudel MS, Tiwari A, Mandal A, Shrestha B, Kafle P, Chaulagai B, et al. Metabolic Syndrome in Patients with Non-alcoholic Fatty Liver Disease: A Community Based Cross-sectional study. Cureus. 2019;11(2). e4099. doi: 10.7759/cureus.4099. [PubMed: 31057993]. [PubMed Central: PMC6476620].

25. Cameron AJ, Magliano DJ, Zimmet PZ, Welborn T, Shaw JE. The metabolic syndrome in Australia: prevalence using four definitions. Diabetes Res Clin Pract. 2007;77(3):471-8. doi: 10.1016/j.diabres.2007.02.002. [PubMed: 17350710].

26. Mazloomzadeh S, Rashidi Khazaghi Z, Mousavinasab N. The Prevalence of Metabolic Syndrome in Iran: A Systematic Review and Metaanalysis. Iran J Public Health. 2018;47(4):473-80. [PubMed: 29900131]. [PubMed Central: PMC5996331].

27. Ervin RB. Prevalence of metabolic syndrome among adults 20 years of age and over, by sex, age, race and ethnicity, and body mass index: United States, 2003-2006. Natl Health Stat Report. 2009;(13):1-7. [PubMed: 19634296].

28. Patton HM, Yates K, Unalp-Arida A, Behling CA, Huang TT, Rosenthal $P$, et al. Association between metabolic syndrome and liver histology among children with nonalcoholic Fatty liver disease. Am J Gastroenterol. 2010;105(9):2093-102. doi: 10.1038/ajg.2010.152. [PubMed: 20372110]. [PubMed Central: PMC3070291].
29. Shabalala SC, Dludla PV, Mabasa L, Kappo AP, Basson AK, Pheiffer $\mathrm{C}$, et al. The effect of adiponectin in the pathogenesis of nonalcoholic fatty liver disease(NAFLD) and the potential role of polyphenols in the modulation of adiponectin signaling. Biomed Pharmacother. 2020;131:110785. doi: 10.1016/j.biopha.2020.110785. [PubMed 33152943].

30. Pafili K, Roden M. Nonalcoholic fatty liver disease (NAFLD) from pathogenesis to treatment concepts in humans. Mol Metab. 2021;50:101122. doi: 10.1016/j.molmet.2020.101122. [PubMed: 33220492]. [PubMed Central: PMC8324683].

31. Azzout-Marniche D, Becard D, Guichard C, Foretz M, Ferre P, Foufelle F. Insulin effects on sterol regulatory-element-binding protein1c (SREBP-1c) transcriptional activity in rat hepatocytes. Biochem J. 2000;350 Pt 2:389-93. [PubMed: 10947952]. [PubMed Central: PMC1221265].

32. da Silva Rosa SC, Nayak N, Caymo AM, Gordon JW. Mechanisms of muscle insulin resistance and the cross-talk with liver and adipose tissue. Physiol Rep. 2020;8(19). e14607. doi: 10.14814/phy2.14607. [PubMed: 33038072]. [PubMed Central: PMC7547588].

33. Caballeria L, Pera G, Auladell MA, Toran P, Munoz L, Miranda D, et al. Prevalence and factors associated with the presence of nonalcoholic fatty liver disease in an adult population in Spain. Eur J Gastroenterol Hepatol. 2010;22(1):24-32. doi: 10.1097/MEG.ob013e32832fcdfo. [PubMed: 19730384].

34. Rector RS, Thyfault JP, Wei Y, Ibdah JA. Non-alcoholic fatty liver disease and the metabolic syndrome: an update. World J Gastroenterol. 2008;14(2):185-92. doi: 10.3748/wjg.14.185. [PubMed: 18186553]. [PubMed Central: PMC2675112] 